\title{
Hierarchical renormalization group fixed points
}

\author{
A. Pordt* \\ Institut für Theoretische Physik I, Universität Münster, \\ Wilhelm-Klemm-Str. 9, D-48149 Münster, Germany
}

November 13, 2018

\begin{abstract}
Hierarchical renormalization group transformations are related to nonassociative algebras. Non-trivial infrared fixed points are shown to be solutions of polynomial equations. At the example of a scalar model in $d(\geq 2)$ dimensions some methods for the solution of these algebraic equations are presented.
\end{abstract}

\section{Introduction}

Wilson's renormalization group (RG) approach [1] is an important tool for the investigation of quantum field theories. The study of the ultraviolet (UV) and infrared (IR) limits correspond to the study of field theories near fixed points of a renormalization group transformation. A trivial fixed point (UV fixed point) corresponds to asymptotically free theories. For these models standard perturbation methods are sufficient. There are other non-trivial RG fixed points (IR fixed points) where a small parameter is lacking. The goal of this paper is to present non-perturbative methods for analyzing models at IR fixed points.

Investigation of quantum field theories at infrared fixed points requires nonperturbative methods. In this paper we want to consider hierarchical RG fixed points.

The hierarchical RG transformation (HRGT) for a scalar field theory is defined in the following. Let $Z: \mathbf{R} \rightarrow \mathbf{R}$ be a (Gaussian-measurable) function (which corresponds to the generating functional of free propagator-amputated Greens functions). $Z$ is also called partition function. Application of the HRGT $\mathcal{R G}$ to the partition function $Z$ yields the effective partition function $\mathcal{R G}(Z): \mathbf{R} \rightarrow \mathbf{R}$ defined by

$$
\mathcal{R G}(Z)(\Psi):=\int d \mu_{\gamma}(\Phi)\left[Z\left(\Phi+L^{1-\frac{d}{2}} \Psi\right)\right]^{L^{d}},
$$

\footnotetext{
*Work supported by the Deutsche Forschungsgemeinschaft under Grant Wi 1280/2-1
} 
where $\Psi \in \mathbf{R}, L \in\{2,3, \ldots\}, \gamma>0$ and the Gaussian measure is defined by

$$
\int d \mu_{\gamma}(\Phi):=\int_{-\infty}^{\infty} d \Phi \exp \left\{-\frac{\Phi^{2}}{2 \gamma}\right\}
$$

The factor $L$ corresponds to the block size of a block spin RG transformation and $\gamma$ corresponds to the fluctuation propagator. The generalization to $N$-component models is straightforward.

We want to find even fixed points $Z^{*}$ of the $\operatorname{HRGT} Z^{*}=\mathcal{R} \mathcal{G}\left(Z^{*}\right)$. Excluding $Z^{*}=0$ or $\infty$, we immediately find the following trivial fixed points. There are the UV fixed point $Z_{U V}(\Psi)=1$ and the high-temperature fixed point $Z_{H T}(\Psi)=$ $L^{\frac{1}{L^{d}-1}} \exp \left\{-\frac{L^{2}-1}{2 \gamma L^{d}} \Psi^{2}\right\}$. For $d \geq 4$ there are only these trivial fixed points. In $2 \leq$ $d<4$ dimensions it turns out that there exists also other non-trivial fixed points which are called infrared fixed points. For a further investigation one introduces the hierarchical RG algebra [3].

\section{Hierarchical renormalization group algebras}

Let $P(\Phi)$ be a polynomial in $\Phi$. Define normal-ordering (Wick-ordering) of $P(\Phi)$ by

$$
: P(\Phi):_{\gamma}:=\exp \left\{-\frac{\gamma}{2} \frac{\partial^{2}}{\partial \Phi^{2}}\right\} P(\Phi)
$$

Introduce structure coefficients $\mathcal{C}_{l}^{m n}$ by the fusion relations

$$
\begin{aligned}
& : \Phi^{2 m}:_{\gamma} \cdot: \Phi^{2 n}:_{\gamma}= \\
& \sum_{l:|m-n| \leq l \leq m+n} \mathcal{C}_{l}^{m n}: \Phi^{2 l}:_{\gamma} .
\end{aligned}
$$

Let $\left\{e_{m}, m=0,1,2, \ldots\right\}$ be the canonical basis of the vector space $\mathbf{R}^{\infty}$. Define a $\times$-product on $\mathbf{R}^{\infty}$ by

$$
e_{m} \times e_{n}:=\sum_{l:|m-n| \leq l \leq m+n} \mathcal{C}_{l}^{m n} e_{l}
$$

Then $\left(\mathbf{R}^{\infty}, \times\right)$ is a commutative and associative algebra with unit element $e_{0}$.

We will identify partition functions $Z: \mathbf{R} \rightarrow \mathbf{R}$ with elements $z=\left(z_{0}, z_{1}, z_{2}, \ldots\right)=$ $\sum_{n=0}^{\infty} z_{n} e_{n}$ of the algebra $\mathbf{R}^{\infty}$ by

$$
Z(\Phi)=\sum_{n=0}^{\infty} \frac{z_{n}}{\gamma^{n}}:\left(\Phi^{2}\right)^{n}:_{\gamma} .
$$

For $z \in \mathbf{R}^{\infty}$ and $\beta \in \mathbf{R}$ define the scaling relation $\mathcal{S}_{\beta}: \mathbf{R}^{\infty} \rightarrow \mathbf{R}^{\infty}$ by $\mathcal{S}_{\beta}(z)_{l}:=\beta^{2 l} z_{l}$. Then one can show that the HRGT eq. (1) - where $\gamma$ is replaced by $\left(1-\beta^{2}\right) \gamma-$ is equivalent to

$$
\mathcal{R G}(z)=\mathcal{S}_{\beta}(\underbrace{z \times \cdots \times z}_{L^{d} \text { factors }})
$$


where $\beta:=L^{1-\frac{d}{2}}$. Let us remark that this relation is only helpful if $|\beta|<1$. This seems to exclude the case $d=2$. We may avoid this problem by a transformation of the HRGT eq. (1). Define a new partition function $F$ by $F:=Z / Z_{H T}$. Then the HRGT of $F$ has the same form as the HRGT eq. (四) of $Z$. Only the coefficient $\gamma$ has to be replaced by $L^{-2} \gamma$ and the scaling parameter $\beta$ by $L^{-2} \beta=L^{-1-\frac{d}{2}}<1$. This method is called "extraction of the high-temperature fixed point".

For the simplest choice $L^{d}=2$ the fixed points $z^{*}$ are solutions of the quadratic equation $z=z \times_{\beta} z:=\mathcal{S}_{\beta}(z \times z)$. The product $\times_{\beta}$, for $\beta \neq 1$, defines a commutative and non-associative algebra $\left(\mathbf{R}^{\infty}, \times_{\beta}\right)$ containing no unit element. The algebra $\left(\mathbf{R}^{\infty}, \times_{\beta}\right)$ is called a hierarchical renormalization group algebra (HRGA). By change of coordinates there are also other HRGA's possible. For example, identify the partition functions $Z: \mathbf{R} \rightarrow \mathbf{R}$ with elements $\widetilde{z}=\left(\widetilde{z}_{0}, \widetilde{z}_{1}, \widetilde{z}_{2}, \ldots\right)=\sum_{n=0}^{\infty} \widetilde{z}_{n} e_{n}$ of the algebra $\mathbf{R}^{\infty}$ by series expansion

$$
Z(\Phi)=\sum_{n=0}^{\infty} \frac{\widetilde{z}_{n}}{\gamma^{n}}\left(\Phi^{2}\right)^{n} .
$$

Then one can show that the HRGT eq. (1) is equivalent to

$$
\mathcal{R G}(\widetilde{z})=\mathcal{S}_{\beta}(\widetilde{z} \star \widetilde{z})=\widetilde{z} \star_{\beta} \widetilde{z}
$$

where the $\star_{\beta}$-product defines the non-associative algebra $\left(\mathbf{R}^{\infty}, \star_{\beta}\right)$ and the corresponding structure coefficients $\mathcal{S}_{l}^{m n}$ can be easily computed [2, 3]. Let us remark that this product cannot be used for cases $L^{d} \neq 2$, since $\left(\mathbf{R}^{\infty}, \star_{\beta}\right)$ is also nonassociative for $\beta=1$.

For periodic fixed points in $d=2$ dimensions one represents the partition function $Z$ with period $T=\frac{\sqrt{\gamma}}{q}$ by

$$
Z(\Phi)=\sum_{m 0-\infty}^{\infty} z_{m} \exp \left\{2 \pi i m \frac{q}{\sqrt{\gamma}} \Phi\right\}
$$

Define a $\circ_{q}$-product by

$$
e_{m} \circ_{q} e_{n}:=\exp \left\{-2 \pi^{2} q^{2}(m+n)^{2}\right\} e_{m+n} .
$$

Then the HRGT eq. (11), for $d=2$ and $L^{2}=2$, is equivalent to

$$
\mathcal{R G}(z)=z \circ_{q} z
$$

The HRGA $\left(\mathbf{R}^{\infty}, \circ_{q}\right)$ is non-associative for $q \neq 0$.

\subsection{Norms}

For estimations and convergence proofs suitable definitions of norms for HRGA's are required. Equipped with these norms the algebras $\mathbf{R}^{\infty}$ become Banach-algebras. 
The norms $\|\cdot\|_{\rho}$, defined below, depend on a parameter $\rho$. If the parameter $\rho$ is large enough, $\rho \geq \rho^{*}$, the norms become algebra-norms, i. e. they obey

$$
\|a \bullet b\|_{\rho} \leq\|a\|_{\rho} \cdot\|b\|_{\rho},
$$

where the $\bullet$-product defines a HRGA. For example, Koch and Wittwer [1] used the following norm for their proof of a non-trivial fixed point in $d=3$ dimensions for the case $L^{d}=2$ and extracting the high-temperature fixed point $\left(2 \beta^{2}<1\right)$

$$
\|z\|_{\rho}^{(1)}:=\sum_{n=0}^{\infty} \sqrt{(2 n) !}\left|z_{n}\right| \rho^{n} .
$$

For the use of estimating coefficients in the $\epsilon$-expansion the following sup-norm is useful [3] $\left(2 \beta^{2}>1\right)$

$$
\|z\|_{\rho}^{(\infty)}:=\sup _{n}\left(n !\left|z_{n}\right| \rho^{n}\right) .
$$

In constructive field theory norms are typically defined by a large field regulator $e^{\rho \Phi^{2}}$

$$
\|z\|_{\rho}^{(c)}:=\sup _{\Phi \in \mathbf{R}}\left|e^{\rho \Phi^{2}} Z(\Phi)\right| .
$$

\section{Methods and Results}

There are several methods to solve the fixed point equation. Beside the method of $\epsilon$-expansion all methods presented here deliver convergent expansions for the fixed points.

The first and most standard one is to iterate the RGT. Define a sequence of partition functions by $z^{(n+1)}:=\mathcal{R} \mathcal{G}\left(z^{(n)}\right)$. One has to choose the start of the RGT $z^{(0)}$ such that $\lim _{n \rightarrow \infty} z^{(n)}=z^{*}$ exists. Then $z^{*}$ is a fixed point.

A second standard method is the $\epsilon$-expansion [5, 21. Take the trivial UV fixed point $z_{U V}=e_{0}$ in $d_{*}$ dimensions. Then one considers the fixed point equation in $d=d_{*}-\epsilon$ dimensions. The fixed point $z^{*}$ and the scaling factor $\beta^{2 l}$ is written as a power series in $\epsilon$. Insertion of these series in the fixed point equation $z=z \times_{\beta} z$ and comparison of the coefficients yields recursive equations for the coefficients of the fixed point in the case of critical dimensions $d_{*}=d_{l}:=\frac{2 l}{l-1}, l \in\{2,3, \ldots\} . \ln Z^{*}(\Phi)$ has the form of an $l$-well.

That $d_{l}$ are the critical dimensions can be seen in the following way. We suppose that for small $\epsilon$ the fixed point $z=e_{0}+r$ is near by the UV fixed point $e_{0}$, i. e. $r$ is small. The fixed point equation in terms of $r$ is $r=2 e_{0} \times_{\beta} r+r \times \times_{\beta} r$. In lowest order $r$ should obey $r \in \operatorname{ker}\left(i d-2 e_{0} \times_{\beta}\right)$. The linear operator $\left(i d-2 e_{0} \times_{\beta}\right)=$ $\operatorname{diag}\left(1-2 \beta^{2 l}, l=0,1, \ldots\right)$ has only a non-trivial kernel if there exists $l$ such that $1=2 \beta^{2 l}$. This is the case for the critical dimensions $d=d_{l}$.

A third method used by Koch and Wittwer [4] is the method of exact betafunctions. For that, one defines a projection operator $\mathrm{P}$ on $\mathbf{R}^{\infty}$ such that

$$
z^{r e l}=z^{r e l}\left(\gamma_{0}, \ldots, \gamma_{N}\right)=\mathrm{P}(z)
$$


depends only on a finite number of parameters $\gamma_{0}, \ldots, \gamma_{N}$ and such that the irrelevant part of $z$ defined by $r:=(1-\mathrm{P})(z)$ can be controlled by standard fixed point theorems. The irrelevant fixed point $r^{*}=r^{*}(\gamma)$ is defined by

$$
r^{*}=H_{\gamma}\left(r^{*}\right):=(\mathbf{1}-\mathrm{P}) \mathcal{R} \mathcal{G}\left(z^{r e l}(\gamma)+r^{*}\right) .
$$

The exact beta-function $B: \mathbf{R}^{N+1} \rightarrow \mathbf{R}^{N+1}$ is defined by

$$
\begin{aligned}
& z^{\text {rel }}\left(B\left(\gamma_{0}, \ldots, \gamma_{N}\right)\right)= \\
& \quad \operatorname{PR\mathcal {G}}\left(z^{r e l}\left(\gamma_{0}, \ldots, \gamma_{N}\right)+r^{*}\left(\gamma_{0}, \ldots, \gamma_{N}\right)\right) .
\end{aligned}
$$

The fixed points $z^{*}=\mathcal{R} \mathcal{G}\left(z^{*}\right)$ are given by $z^{*}=z^{\text {rel }}\left(\gamma^{*}\right)+r^{*}\left(\gamma^{*}\right)$, where $\gamma^{*}$ is the fixed point of the exact beta-function $B, B\left(\gamma^{*}\right)=\gamma^{*}$. For the HRGT one can use the projection operator

$$
\mathrm{P}(z):=\left(z_{0}, \ldots, z_{N}, 0,0, \ldots\right)
$$

and the beta-function technique works for $N \geq N_{0}=O(1)(=7)$.

A fourth method is to replace the fixed point equation by a minimizationproblem. Define an energy-function $E(z)$ for $z \in \mathbf{R}^{\infty}$ by

$$
E(z):=\sum_{l=0}^{\infty}\left(z_{l}-\left(z \times_{\beta} z\right)_{l}\right)^{2} \geq 0 .
$$

Then $z=z \times_{\beta} z$ is equivalent to $E(z)=0$.

A fifth method is to solve the equation $z \times_{\beta} z-z=0$ directly by Newton's method [2].

A sixth method is to truncate the infinite system of quadratic equations by setting $z_{l}=0$ if $l>N$ and solve the finite system directly by means of Groebner bases.

For convergence proofs and numerical calculations one has to truncate the infinite system. One can show that there exists a solution of the truncated fixed point equation $z^{(N)}$ such that the $\operatorname{limit}_{\lim _{N \rightarrow \infty}} z^{(N)}=z^{*}$ exists.

\section{References}

[1] K. G. Wilson, Physica 73 (1974) 119; K. G. Wilson, J. Kogut, Phys. Rep. C12 (1974) 12 .

[2] K. Pinn, A. Pordt, C. Wieczerkowski, Journ. Stat. Phys. 77 (1994) 977.

[3] A. Pordt, C. Wieczerkowski, MS-TPI-94-04.

[4] H. Koch, P. Wittwer, Commun. Math. Phys. 106 (1986) 495; Commun. Math. Phys. 138 (1991) 537.

[5] P. Collet, J.-P. Eckmann, Commun. Math. Phys. 55 (1977) 67. 